\title{
émulations
}

\section{Par la tête et par le cour}

\section{En souvenir de l'enseignement d'Enric Porqueres i Gené}

\author{
Noémie Merleau-Ponty \\ Émulations - Revue de sciences sociales \\ 2019, n 32, «Aux frontières de la parenté. Un éclairage par les marges ».
}

Article disponible à l'adresse suivante

https://ojs.uclouvain.be/index.php/emulations/article/view/merleauponty

\section{Pour citer cet article}

Noémie Merleau-Ponty, «Par la tête et par le cœur. En souvenir de l'enseignement d’Enric Porqueres i Gené », Émulations, n³2, Mise en ligne le 20 avril 2020. DOI : 10.14428/emulations.032.02

Distribution électronique : Université catholique de Louvain (Belgique) : ojs.uclouvain.be

(C) Cet article est mis à disposition selon les termes de la Licence Creative Commons Attribution, Pas d'Utilisation Commerciale 4.0 International. http://creativecommons.org/licenses/by-nc/4.0/

Éditeur : Émulations - Revue de sciences sociales / Presses universitaires de Louvain https://ojs.uclouvain.be/index.php/emulations

ISSN électronique : 1784-5734

$\frac{\text { PUL PRESSES }}{\text { UNIVERSITAIRES }}$ 


\section{Par la tête et par le cœur En souvenir de l'enseignement d'Enric Porqueres i Gené}

Noémie Merleau-Ponty ${ }^{1}$

Le travail de deuil n'est pas facile. Il faut le dire, il faut l'écrire, pour pouvoir le commencer. Je tourne autour de ce texte, je sens ma pensée et mes émotions fuir, nourrir la page blanche de mots fugitifs pour ne pas avoir à se confronter, encore, à la disparition.

Enric Porqueres i Gené (1962-2018) enseigna l'anthropologie de la parenté à l'École des hautes études en sciences sociales (EHESS) vingt-deux années durant. Je fis sa connaissance alors que j'étais étudiante en Master et m'inscrivis en thèse de doctorat sous sa direction en 2011.

Écrire inscrit une forme de permanence, fixe quelques signes entrelacés contre l'oubli de la disparition et, dans cette fixation, poursuit le travail de transmission. S'il s'agit de cela, alors d'accord, j'accepte de me replonger dans le souvenir, d'ouvrir la mémoire personnelle pour la rendre un tant soit peu publique. Je tiens à remercier le groupe de recherche EnCoRe (Engendrement, Corps, Relation), qui a ouvert au sein de ce numéro thématique un espace dans lequel un sillon peut suggérer une trace. Cette trace, celleci, concerne l'art de faire de l'anthropologie " par la tête » et "par le cœur ", comme le dit si poétiquement Clara Hyunjung Lee, qui a également réalisé une thèse sous la direction de notre cher disparu.

Cet hommage souhaite rappeler la vivacité d'un enseignement, rendre compte d'un séminaire dans lequel s'est déployée «la recherche en train de se faire » dirait l'EHESS. Pour Enric Porqueres i Gené, cette recherche ne pouvait se prémunir d'allier théorie et pratique. Toutes celles et tous ceux qui peuvent en témoigner le soulignent. Enric Porqueres i Gené, ou Enric Porqueres, ou Enric, ou Monsieur Porqueres, ou Monsieur Porqueres i Gené, a tenu des séminaires de recherche et d'enseignement à l'EHESS, avec un art intellectuel et sensible que je ne perçus pas tout de suite, alors que j'en bénéficiais, comme beaucoup d'autres étudiantes et étudiants.

Enric fut le seul anthropologue de l'EHESS à faire dialoguer anthropologie de la parenté, biotechnologies de la reproduction et cosmologies embryonnaires non naturalistes, puisant là dans son approche historique de l'Europe, dans des ethnographies classiques, ou dans la philosophie. Il introduisit à l'École les débats britanniques et américains, en les faisant dialoguer avec l'anthropologie française des substances de la parenté. Il ouvrit cet espace de recherche qu'Éric Fassin appelait de ses vœux en 2002.

Les défis contemporains de la parenté (2009a), ouvrage collectif qu'Enric Porqueres i Gené dirigea, permit d'établir un champ de recherche fédérateur. Son séminaire fut

\footnotetext{
${ }^{1}$ Centre national de la recherche scientifique, IRIS, France.
} 
aussi un lieu où se consolidèrent de nombreux projets à ce sujet. L'enseignement d'Enric critiqua sans relâche les distinctions telles que le Nord et le Sud ou l'Occident et le reste du monde. Toujours extrêmement précis dans son approche ethnographique, il n'en suivit pas moins un compas fidèle à l'ambition universaliste de l'anthropologie française. Avec une approche historique et dans un contexte européen, ses travaux sur la personne commencèrent par montrer comment l'individuation ne peut se comprendre sans l'analyse des relations de parenté qui sont tissées par les substances corporelles. Entre minutie descriptive et vaste géopolitique anthropologique, il travailla sans relâche la dialectique relation/individuation, discutant en cela avec les travaux de Marilyn Strathern, issus de ses enquêtes au Royaume-Uni et en Mélanésie. C'est en effet dans son séminaire que plusieurs personnes lurent et discutèrent des passages d'After Nature (1992a) pour la première fois.

La relecture qu'il fit de certaines ethnographies classiques de l'anthropologie permit de mettre à jour des « socles de subjectivité » de la personne, singularisée dans la conception, à travers, par exemple, un souhait quant à son destin, ou encore l'élection d'une femme en qui se développer (Porqueres i Gené, 2014). Il montra ainsi comment les êtres humains prénataux, sans être des personnes ou des individus à part entière, sont cependant dotés d'éléments qui les singularisent. Pour ce faire, il fit référence à des textes dédiés à l'Afrique de l'Ouest, au Pacifique (Australie et îles Trobriand), à la zone arctique, ou encore aux traités médicaux de la médecine ayurvédique indienne. Il analysa ces socles de subjectivité sans évacuer la matérialité des corps et leur porosité relationnelle liant personnes et éléments cosmiques (Porqueres i Gené, 2009a). L'analyse qu'il fit de la parenté chrétienne et de la Una Caro en est un exemple frappant (Porqueres i Gené, 2000, 2015 ; Porqueres i Gené, Wilgaux, 2009).

Ses travaux sur la crainte de l'inceste permirent également de penser les dons de sperme dans une perspective historique et de relativiser la nouveauté des technologies reproductives qui mettraient en avant un embryon-individu, icône de l'humanité (Franklin, 1993 ; Porqueres i Gené, 2004, 2009b ; Strathern, 1992a, 1992b). L'embryon ne peut pas être compris dans une dimension strictement individuelle, comme les images prénatales pourraient le suggérer à propos du fœtus distingué seul sur le fond noir des échographies. Il affirmait ainsi que " nous ne nous trouvons pas dans un monde post-relationnel formé par de purs individus, pas plus que la sacralité juridique de l'embryon, sujet de droit, n'est le fait de notre irréductible différence supposée » (Porqueres i Gené, 2004 : 149). Cette perspective fut accompagnée par un jeu analytique constant entre une conception de la parenté comme descent (« normes juridiques agençant les rapports entre les groupes de filiation en place»), et comme kinship (« représentations natives sur la nature des liens de parenté unissant des individus précis ») (Porqueres i Gené, 2014 : 18).

Les liens qu'il établit entre parenté chrétienne et contemporanéité reproductive lui permirent de travailler avec la sociologue Séverine Mathieu. Ils menèrent ensemble un séminaire sur la norme catholique à propos des biotechnologies de la procréation 
et ont préparé un ouvrage collectif traitant des embryons. Ce texte en cours de publication est un témoignage du séminaire Embryon, personne et parenté, tenu entre les années 2012 et 2017.

Le dernier grand chantier d'Enric Porqueres i Gené, resté inachevé, consista à identifier la présence d'un " je » narratif, supposé nécessaire à la fabrique de la parenté dans des embryologies non européennes. Le séminaire qu'il avait prévu de diriger en 2018-2019 avait d'ailleurs pour titre La personne : entre le corps de la parenté et la langue de l'agentivité.

L'exigence, la bienveillance et la modestie qui faisaient loi dans les séminaires d'Enric permirent à de nombreuses personnes de réfléchir avec de grands textes, aussi ardus soient-ils. Chaque personne présente avait l'occasion de s'exprimer pleinement. Les encouragements, l'enthousiasme, la curiosité réelle d’Enric pour les analyses des séminaristes permirent des échanges très riches. Il prenait des notes, se perdait quelques instants dans une réflexion intérieure, revenait à la discussion, opposait parfois une critique aimable, subtile, de celles qui permettent à l'enseigné·e la malléabilité nécessaire pour une remise en cause génératrice de progrès.

Son accompagnement et son sens de la transmission ont porté leurs fruits, sous de multiples formes, mêlant sans cesse le personnel et le professionnel. Il y avait encore beaucoup à chercher et à transmettre. Grâce à son enseignement, nul doute que d'autres sillons sont déjà au travail, comme ce numéro spécial le démontre.

\section{Bibliographie}

FASSIN E. (2002), «La nature de la maternité. Pour une anthropologie de la reproduction », Journal des anthropologues, $\mathrm{n}^{\circ}$ 88-89, p. 103-122.

FrankLIN S. (1993), « Making representations: the parliamentary debate on the Human Fertilisation and Embryology Act », in J. Edwards, S. Franklin, E. Hirsch, France Price, M. StRATHERn (dir.), Technologies of procreation. Kinship in the age of assisted conception, Manchester, Manchester University Press, p. 96-131.

Porqueres i Gené E. (2000), « Cognatisme et voies du sang », L’Homme, n 154-155, p. 335356.

Porqueres i Gené E. (2004), "Individu et parenté. Individuation de l'embryon », in F. Héritier, M. Xanthakou (dir.), Corps et affects, Paris, France, Odile Jacob, p. 139-150.

Porqueres i Gené E. (2009a), Défis contemporains de la parenté, Paris, France, Éditions de l’École des hautes études en sciences sociales.

PoRQueres I GenÉ E. (2009b), « Individu et personne à la lumière des nouveaux contextes de la parenté », in E. Porqueres i Gené (dir.), Défis contemporains de la parenté, Paris, Éditions de l'École des hautes études en sciences sociales, p. 225-250.

Porqueres i Gené E. (2014), « Personne et parenté », L’Homme, vol. 210, nº 2, p. $17-42$. 
Porqueres i GenÉ E. (2015), Individu, personne et parenté en Europe, Éditions de la Maison des sciences de l'homme.

Porqueres i Gené E., wilgaux J. (2009), " Incest, embodiment, genes and kinship », in J. Edwards, C. SAlazAr (dir.), European kinship in the age of biotechnology, Berghahn Books, p. 112-127.

StRathern M. (1992a), After nature: English kinship in the late twentieth century, Cambridge, Cambridge University Press.

StRATHERN M. (1992b), Reproducing the future: essays on anthropology, kinship and the new reproductive technologies, Manchester, Manchester University Press. 